\title{
Spectrum of Lesions of Gall Bladder: Experience of Five Years at Tertiary Care Hospital
}

\author{
Milind Patil, Mangesh Machindra Londhe*, Sangeeta Margam and Javed Iqbal \\ Department of Pathology, Lokmanya Tilak Municipal Medical College and Lokmanya Tilak Municipal General Hospital, \\ Sion, Mumbai Maharashtra, India.
}

\begin{abstract}
Background: The histopathological spectrum of gallbladder lesions is extremely variable and its frequency in cholecystectomies is not clear. This spectrum varies from non- neoplastic lesions like cholecystitis, gall stones, cholesterolosis, hyperplasia to neoplastic disorders. As there is limited literature available especially from this part of India, the aim of this study is to find incidences of various lesions of gall bladder in a tertiary care hospital.

Methods: Five years retrospective and prospective analysis of 1096 cholecystectomies was carried out. Relevant clinical details of patient were noted down. Cholecystectomy specimens were studied for gross and microscopic examination after fixation of specimen with $10 \%$ formalin and microscopically examining $\mathrm{H} \&$ E slides.

Results: Overall,1096 cases of cholecystectomies were analysed which revealed female preponderance with peak incidence of nonneoplastic lesion in 4th decade and neoplastic lesions in 5th decade. Gall stones were seen in $52 \%$ of cases. Incidence of various lesions was chronic cholecystitis $93.1 \%$, acute cholecystitis $2.91 \%$, chronic follicular cholecystitis $0.72 \%$, xanthogranulomatous cholecystitis $1.1 \%$, cholesterolosis $0.46 \%$, eosinophilic cholecystitis $0.18 \%$, and carcinoma of gall bladder $1.1 \%$.

Conclusion: Present study reveals that gall bladder diseases havediversified lesions including non-neoplastic and neoplastic with different clinical implications. Histopathology plays pivotal role in arriving at final diagnosis thus helping the patient management. It also enables study of detail incidences andhelps to differentiate neoplastic from non-neoplastic lesions which have similar clinical presentation.
\end{abstract}

Keywords: Gall Bladder Lesions, Cholelithiasis, Cholecystitis, Cholesterolosis, Carcinoma Gall Bladder.

\section{Introduction}

The lesions in gall bladder show a vast diversity with similar clinical presentation. Gallstones are found to be the major etiologic factor of gallbladder lesions, the spectrum of which ranges from various types of cholecystistis, cholesterolosis to hyperplasia, metaplasia, dysplasia and fatal malignant transformation. Gallstones are formed mainly due to metabolic problems of hepato-biliary system which is attributed to the increased intake of fatty, high calorie diet and increased consumption of alcohol. ${ }^{[1]}$ It is a common health problem worldwide but its prevalence varies country to country being $2-29 \%$ in India and also varies with age, sex, and ethnic group. ${ }^{[1]}$

Gall bladder diseases are not uncommon and are major reason for morbidity and mortality in our country. The patients remain asymptomatic for many years which partly attributes to incidental findings of many lesions on histopathology including malignancy. Further various findings like metaplasia, hyperplasia, Rokitansky Ashoff sinuses etc. are ignored during histopathology reporting or clubbed together in the form of chronic cholecystitis along with chronic calculus lesions thus hampering the exact determination of their prevalence and prognosis. Cholecystectomy remains the treatment of choice for all gallbladder lesions.

Gallbladder carcinoma (GBC) is a rare condition and as per ICMR report on consensus on management of GBC, the incidence is reported to be $7 / 100,000 \cdot{ }^{[2]} \mathrm{It}$ is commonly diagnosed as an incidental histological finding following cholecystectomy for gallstone disease thus worsening the prognosis. Thus early and accurate histopathological diagnosis of gall bladder lesions may improvise the patient management and prognosis.

Present study was conducted to analyze the histopathological changes associated with the gallbladder disorders in order to ascertain the incidence, prevalence, distribution as well as the histomorphologic spectrum of gall bladder lesions.

\section{Materials and methods}

A 5 years (from January 2004 to December 2008) retrospective and prospective analysis of 1096 cholecystectomy specimen received in surgical pathology department was done. Cholecystectomies received were opened longitudinally, examined for gross features like 
size, colour, external surface, mucosal abnormality and presence of stones and then fixed overnight in $10 \%$ formalin. Lymph nodes if any were dissected. Three sections from wall, body, fundus and neck were taken after fixation. Abnormal mucosa was extensively sampled. Specimens were processed with routine procedure and stained with Haematoxylin and Eosin staining. Special stains like Alcian blue- PAS at pH 2.5 to demonstrate intestinal metaplasia were employed. Surgical records were retrieved for clinical data like age, sex, relevant clinical features. A thorough analysis of clinicopathological correlation was done.

\section{Results}

Over a period of five years, 1096 cholecystectomies were received. Overall female preponderance $(76 \%)$ was seen with male: female ratio being 1:3.2.The age ranged from 8 to 80 years with maximum cases being in $31-40$ years.

Highest incidence of all the gall bladder lesions including malignancy was seen in $4^{\text {th }}$ decade. Chronic right hypochondriac pain was the most common clinical symptom (65\%) followed by vomitting.
Gross morphology revealed a higher percentage of calculous (52\%) than acalculous cholecystectomies (48\%). Amongst the calculus cholecystectomies, highest number of cases was of mixed stones which constituted 57\%, followed by cholesterol stones $12 \%$ and pigment stones $6 \%$. Gall stones were more commonly associated with females $(66 \%)$ than males $(34 \%)$.

On histopathological examination the most common lesion encountered was chronic cholecystitis (93.1\%) followed by acute cholecystitis (2.91\%), xanthogranulomatous cholecystitis (XGC,1.1\%), adenocarcinoma (1.09\%), chronic follicular cholecystitis $(0.72 \%)$, cholesterolosis (0.45\%), adenomyomatous hyperplasia (0.27\%), eosinophilic cholecystitis $(0.18 \%)$ and tuberculous cholecystitis $(0.09 \%)$. Among the epithelial abnormalities, gastric metaplasia was the most common followed by intestinal metaplasia, hyperplasia and malignancy. All malignancies were adenocarcinomas and had no clinical or radiological evidence of the same.

Table 1: Agewise distribution.

\begin{tabular}{|c|c|c|c|c|c|c|c|c|c|c|}
\hline Lesion & $<10$ & $11-20$ & $21-30$ & $31-40$ & $41-50$ & $51-60$ & $61-70$ & $71-80$ & $>80$ & Age Not Mentioned \\
\hline Acute Cholecystitis & 1 & 4 & 8 & 12 & 6 & 3 & 0 & 0 & 0 & 0 \\
\hline Chronic Cholecystitis & 0 & 35 & 214 & 283 & 202 & 152 & 37 & 21 & 0 & 75 \\
\hline \multicolumn{11}{|l|}{-Morphologic } \\
\hline -Chronicfollicular Cholecystitis & 0 & 0 & 0 & 1 & 3 & 1 & 2 & 0 & 0 & 1 \\
\hline -Eosinophillic Cholecystitis & 0 & 0 & 0 & 0 & 1 & 1 & 0 & 0 & 0 & 0 \\
\hline $\begin{array}{l}\text {-Xanthogranulomatous } \\
\text { cholecystitis }\end{array}$ & 0 & 0 & 0 & 8 & 3 & 1 & 0 & 0 & 0 & 0 \\
\hline -Adenomatous Hyperplasia & 0 & 1 & 0 & 1 & 0 & 0 & 0 & 0 & 0 & 1 \\
\hline -Cholesterolosis & 0 & 1 & 0 & 1 & 1 & 0 & 2 & 0 & 0 & 0 \\
\hline -Tuberculous cholecystitis & 0 & 0 & 0 & 0 & 1 & 0 & 0 & 0 & 0 & 0 \\
\hline Adenocarcinoma & 0 & 0 & 0 & 3 & 6 & 1 & 1 & 0 & 0 & 0 \\
\hline -Papillary Adenocarcinoma & 0 & 0 & 0 & 0 & 0 & 1 & 0 & 0 & 0 & 0 \\
\hline -colloid carcinoma & 0 & 0 & 0 & 0 & 0 & 0 & 0 & 0 & 0 & 0 \\
\hline
\end{tabular}

Table 2: Clinico- pathological co-relation.

\begin{tabular}{|l|c|c|c|}
\hline Clinical Features & Acute Cholecystitis & Chronic Cholecystitis & Malignancy \\
\hline Chronic pain in right hypochondria & 10 & 701 & 3 \\
\hline Acute Pain in right hypochondria & 16 & 108 & 7 \\
\hline Jaundice & 0 & 96 & 3 \\
\hline Dyspepsia & 2 & 75 & 1 \\
\hline Vomiting & 12 & 180 & 3 \\
\hline Nausea & 8 & 85 & 0 \\
\hline Fever & 9 & 19 & 0 \\
\hline Lump in abdomen & 0 & 212 & 2 \\
\hline Clinical details not available & 6 & & \\
\hline
\end{tabular}


Table 3: Incidence and sexwise distribution of epithelial abnormalities.

\begin{tabular}{|l|c|c|c|}
\hline Epithelial abnormalities & Incidence & Males & Females \\
\hline Gastric metaplasia & $21 \%$ & $22 \%$ & $78 \%$ \\
\hline Intestinal metaplasia & $17 \%$ & $32 \%$ & $68 \%$ \\
\hline Hyperplasia & $8 \%$ & $24 \%$ & $76 \%$ \\
\hline Malignancy & $1.1 \%$ & $0 \%$ & $100 \%$ \\
\hline
\end{tabular}

\section{Discussion}

Diseases of the gall bladder are common in our country, but only cholelithiasis and cholecystitis were commonly reported .Chronic cholecystitis with associated disorders and other specific histopathological entities were categorised under broad term chronic cholecystitis. Hence detailed incidences of histopathological reporting of these in Indian scenario may not be widely available.

In this study, majority cases were females with male:female ratio being 1:3.2. This female preponderance has also been reported by other studies. The highest incidence of these lesions was seen in between 31-40 years, similar to Siddiqui et al ${ }^{[3]}$ while Devi Beena et al found it more common in $41-50$ years. ${ }^{[4]}$

Most common symptom was pain in right hypochondriac region followed by vomiting similar to other studies and is well documented in literature. None of the cases had any clinical or radiological evidence of malignancy.

On gross morphological analysis, calculous cholecystectomies constituted higher percentage (52\%). Dattal D S et al have also reported higher percentage of $91.8 \%$, which however is much more. ${ }^{[1]}$ Thus etiologies other than gall stones needs to be evaluated. Majority of stones in our study were mixed type (57\%) [Fig. 2A] which was comparable to study of Goyal et al $(68 \%) \cdot{ }^{[5]}$ In contrast pigmented stones were more common in studies conducted by Devi Beena et al ${ }^{[4]}$ and Selvi et al. ${ }^{[6]}$

The most common histopathological finding in our study was chronic cholecystitis (98\%). Similar studies have also reported the same finding. Acute cholecystitis consisted of $2.91 \%$. Most of these patients had fever and nausea.

Incidence of Chronic follicular cholecystitis was $0.725 \%$, which is consistent with findings of R.L.Estrado et al, ${ }^{[12]}$ who reported incidence of $0.4 \%$. H.Mohan et al ${ }^{[13]}$ reported incidence of $2.3 \%$ in his series of 1100 cholecystectomies. This is one of the variant of chronic cholecystitis with presence of conspicuous germinal centres in the lamina as well as muscularis and should not be confused with Non-
Hodgkin's lymphoma especially when lymphoid infiltrate is diffuse[Fig. 1B].

Incidence of cholesterolosis was $0.45 \%$. S.P. Sharma et al, ${ }^{[14]}$ reported similar incidence of $1.7 \%$, whereas H.Mohan et $\mathrm{al}^{[13]}$ reported higher incidence i.e. $10 \%$. On morphology mucosa revealed linear yellow streaks against fiery red mucosa, called as strawberry gall bladder. Microscopy revealed accumulation of lipid laden macrophages accumulated at tips of villi [Fig. 1C].

Incidence of XGC was $1.1 \%$ with maximum cases seen in 31 40 years with female predominance. Gilberto et al reported $1.465 \%,{ }^{[15]}$ similar to our study. However H.Mohan et al ${ }^{[13]}$ reported higher incidence of $2.6 \%$. However XGC is seen commonly in $5^{\text {th }}$ and $6^{\text {th }}$ decade which is in contrast to our study. The mean age of 43 years and female preponderance reported by Dixit et al is comparable. ${ }^{[16]}$ XGC grossly mimics carcinoma of gall bladder as it causes thickening of the wall. Association of XGC and GBC is well known but in our study such association was not seen.

Eosinophilic cholecystitis is rare with incidence in our study being $0.18 \%$ similar to H.Mohan et al ${ }^{[13]}(0.6 \%)$. Fox and Main Warning et al, ${ }^{[17]}$ reported incidence of $0.48 \%$ and concluded that eosinophils were an unusual cellular response to the presence of gall stones. But Dabs DJ ${ }^{15}$ found out of 14 cases with very prominent eosinophils were acalculous cholecystitis similar to both the cases in our study suggesting that inflammatory response may be idiopathic, or attributed to presence of altered bile or bile stasis. ${ }^{[18]}$ Massive infiltration by eosinophils forming sheets should be taken into consideration rather than just presence of eosinophils in an otherwise polymorphic infiltrate [Fig. 1D].

Epithelial metaplastic changes like gastric and intestinal were observed in $21 \%$ and $16.9 \%$ of cases respectively [Fig. 2C and 2D]. This incidence is low in present study as limited number of gall bladder sections were studied, but extensive sampling would have detected focal and patchy metaplastic areas. Duarte et al, ${ }^{[19]}$ reported incidence of $62.7 \%$ of gastric and $27.2 \%$ of intestinal metaplasia. 
Incidence of adenomyomatous hyperplasia was $0.27 \%$ which varies from as low as 0.65 to 33.35 of all gall bladder diseases depending on investigative modality like radiological or surgical. ${ }^{[20]}$ Histology revealed proliferation of both epithelial and smooth muscles which may mimic malignancy.

Despite of high prevalence of tuberculosis of gastrointestinal tract tuberculous involvement of gall bladder is very rare. We came across single case in 45 years old lady, presented with right hypochondriac pain, small fibrosed GB with thickened wall and histopathology revealed usual granulomatous inflammation.

Carcinoma of gall bladder is rare but lethal disease. Incidence of Gall bladder carcinoma shows considerable variation in geography, gender and racial differences. Further incidence varies in different part of world and also differs amongst different ethnic groups within same country. Within India incidence varies within Indian population, high incidence in Northern India i.e. 4.5 per 100,000 for males and $10.1 \%$ for females, as opposed to low incidence in south India i.e. 1.2 per 100,000 for males and $0.9 \%$ per 100,000 for females is reported by population based cancer registry Delhi. ${ }^{[21]}$ We reported incidence of $1.1 \%$ which is comparable to study of H.Mohan et al ${ }^{[13]}(1.09 \%)$ while S.P.Sharma et al ${ }^{[14]}$ reported high incidence of $17 \%$, which was attributed to geographic, ethic or dietary factors. In our study, most cases were observed in $5^{\text {th }}$ decade. Moreover, all cases were females. As per literature most patients presents in $6^{\text {th }}$ and $7^{\text {th }}$ decades with female preponderance. Pandey et al ${ }^{[22]}$ reported 98 total gall bladder cancers, 24 male and 74 females with age incidence of 70-75 years. Also Esenda $\breve{G} l i \mathrm{G}$ et al reported incidence of GB adenocarcinoma as $0.4 \%$ which was lower than our study. ${ }^{[23]}$ Presence of gall stones is important factor for development of GBC. We found 6 out 12 cases associated with gall stones. Mohan H. et al reported $100 \%$ association indicating its association with cancer. Combination of factors such as chronic irritation, infection, local metabolic dysfunction, bile stasis and individual host susceptibility might be responsible for carcinoma. Most common histological type was adenocarcinoma, moderately differentiated was commonest and a single case of papillary adenocarcinoma was seen. Other histological types were not seen in present study. Study of adjacent mucosa is essential which may reveal metaplasia, hyperplasia, dysplasia which is pathway for carcinogenesis.

Table 4: Comparison of age in various studies.

\begin{tabular}{|c|c|c|c|c|c|c|}
\hline Age group & Present study & $\begin{array}{l}\text { Devi beena et } \\
\text { al }(2017)^{[4]}\end{array}$ & $\begin{array}{l}\text { Awasthi et al } \\
(2015)^{[7]}\end{array}$ & $\begin{array}{l}\text { Memon et al } \\
(2011)^{[8]}\end{array}$ & $\begin{array}{l}\text { Nigam et al } \\
(2013)^{[9]}\end{array}$ & $\begin{array}{l}\text { Narendra et al } \\
(2015)^{[10]}\end{array}$ \\
\hline $11-20$ & $3.74 \%$ & $1.5 \%$ & $2.9 \%$ & $0.0 \%$ & $0.0 \%$ & $10 \%$ \\
\hline $21-30$ & $20.25 \%$ & $12.5 \%$ & $18.2 \%$ & $13.8 \%$ & $9.6 \%$ & $22 \%$ \\
\hline $31-40$ & $28.20 \%$ & $23 \%$ & $23.5 \%$ & $31.9 \%$ & $20.38 \%$ & $37 \%$ \\
\hline $41-50$ & $20.34 \%$ & $26 \%$ & $27.2 \%$ & $31.9 \%$ & $41.92 \%$ & $15 \%$ \\
\hline $51-60$ & $14.60 \%$ & $22 \%$ & $19.5 \%$ & $20.5 \%$ & $18.46 \%$ & $08 \%$ \\
\hline$>60$ & $13.17 \%$ & $15 \%$ & $8.7 \%$ & $1.7 \%$ & $9.61 \%$ & $08 \%$ \\
\hline
\end{tabular}

Table 5: Comparison of histopathological (HP) lesions in various studies.

\begin{tabular}{|l|l|l|l|l|l|}
\hline HP lesions & Present study & $\begin{array}{l}\text { Devi Beena et al } \\
(\mathbf{2 0 1 7})^{[4]}\end{array}$ & $\begin{array}{l}\text { Goyal et al } \\
(\mathbf{2 0 1 4})^{[5]}\end{array}$ & $\begin{array}{l}\text { Arathi et al } \\
(\mathbf{2 0 1 3})^{[11]}\end{array}$ & $\begin{array}{l}\text { Selvi et al } \\
(\mathbf{2 0 1 1})^{[6]}\end{array}$ \\
\hline Chronic cholecystitis & $98 \%$ & $82 \%$ & $57.8 \%$ & $86.3 \%$ & $87 \%$ \\
\hline Acute cholecystitis & $2.9 \%$ & $3.5 \%$ & $9.5 \%$ & $8.4 \%$ & $2.5 \%$ \\
\hline XGC & $1.1 \%$ & $1 \%$ & $2.5 \%$ & $3.8 \%$ & $1.2 \%$ \\
\hline Cholesterolosis & $0.45 \%$ & $2.5 \%$ & $3.9 \%$ & $2.5 \%$ \\
\hline
\end{tabular}




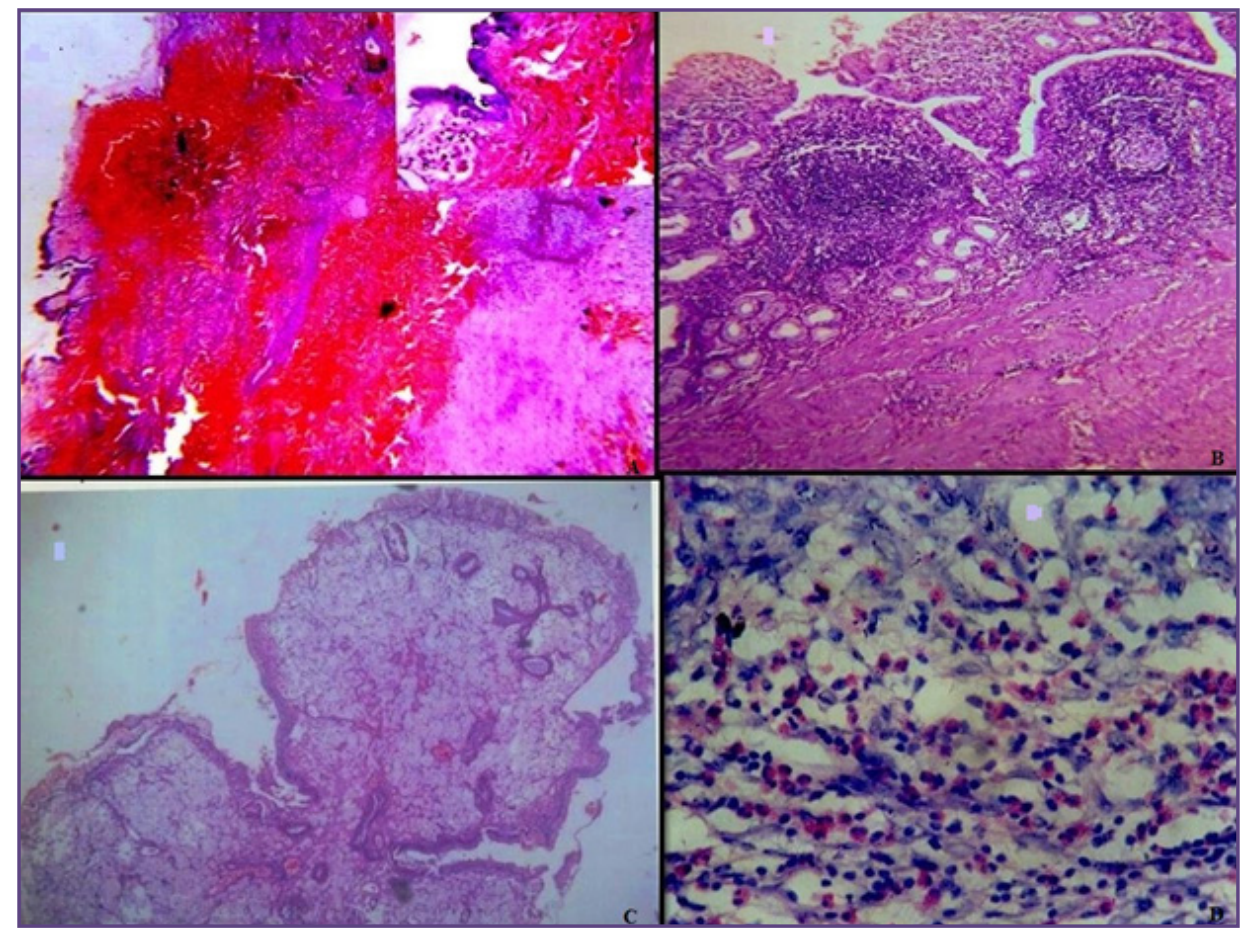

Fig. 1A: acute cholecystitis showing haemorrhage $(H \& E \times 100)$ with inset showing regenerating mucosa $(H \& E \times 400)$. 1B: chronic follicular cholecystitis showing lymphoid follicular germinal centres in lamina propria with gastric metaplasia (H\&E×100). 1C: cholesterolosis showing numerous foamy macrophages in the lamina propria (H\&E×100). 1D:eosinophilic cholecystitis showing transmural eosinophils ( H\&E×100).

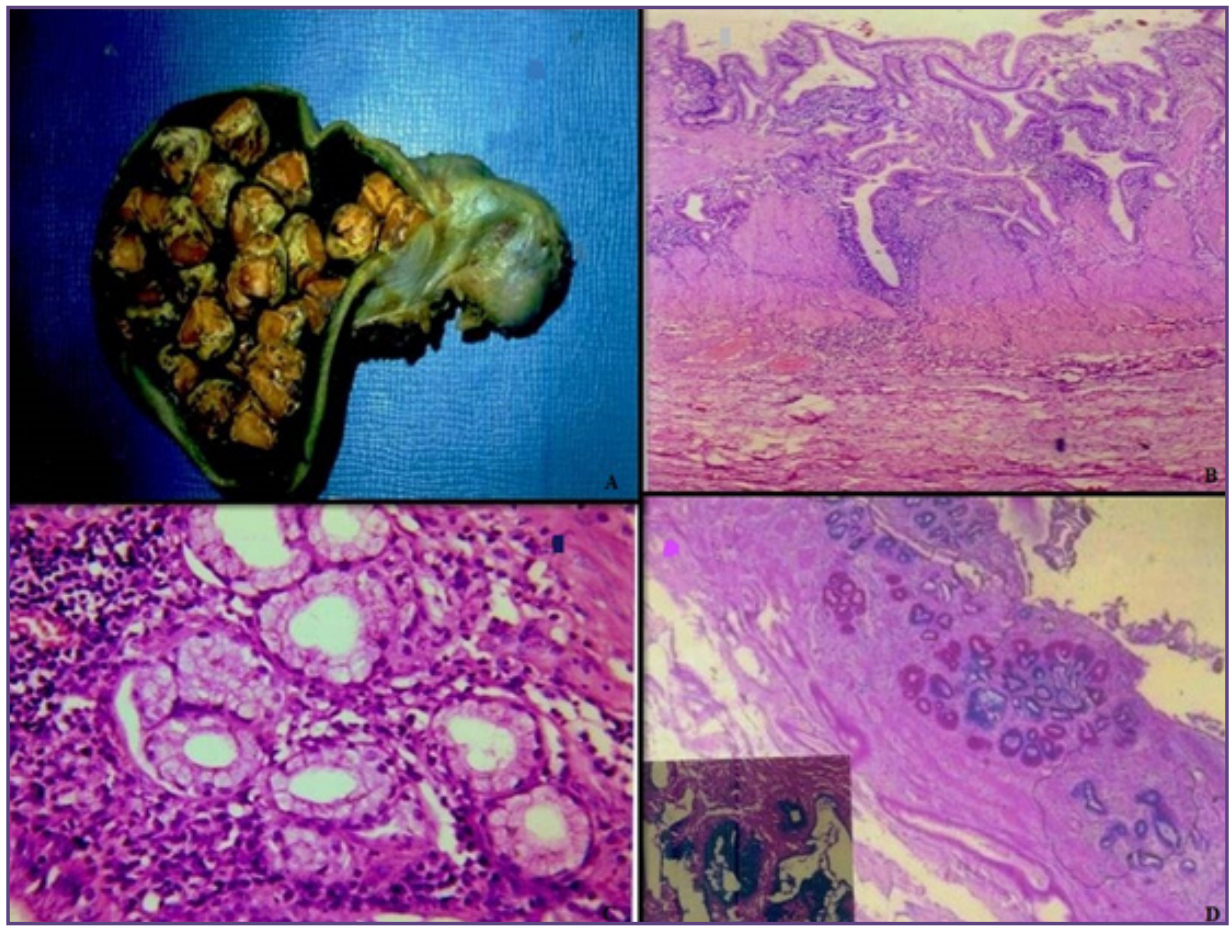

Fig. 2A: GB showing mixed stones. 2B: Rokitansky-Aschoff sinuses (H\&E× 100). 2C: Gastric metaplasia (H\&E×400). 2D:Intestinal metaplasia showing goblet cell stained with alcian blue- PAS(H\&E×100) with inset showing the same at $400 X$.. 


\section{Conclusion}

Gall bladder diseases are common occurrence, especially non- neoplastic lesions. Exact incidence of various lesions in Indian population can be determined by large population based large studies. Also etiological factors other than gallstone, younger age of presentation and increasing incidence in males needs to evaluated. One needs to identify clinical mimickers of gall bladder carcinoma and precursor lesions like metaplasia, which is possible only by histopathological evaluation.

\section{References}

1. Dattal DS, Kaushik R, Gulati A, Sharma VK. Morphological spectrum of gall bladder lesions and their correlation with cholelithiasis. Int J Res Med Sci 2017;5:840-6.

2. Shukla HS, Sirohi B, Behari A et al. ICMR consensus document for the management of gall bladder cancer. Indian Journal of Medical and Paediatric Oncology 2015; 36(2):79-84.

3. Siddiqui FG, Memon AA and Ahmad. Routine histopathology of gallbladder after elective cholecystectomy for gallstones: waste of resources or a justified act? BMC Surgery. 2013; $13: 26$.

4. Devi B, Shetty J, Bose V. Histopathological Spectrum of Diseases in Gallbladder. National Journal of Laboratory Medicine. 2017; 6(4): 6-9.

5. Goyal S, Singla S, Duhan A. Correlation between gallstones characteristics and gallbladder mucosal changes: A retrospective study of 313 patients. Clin Cancer Investig J. 2014;3:157-61.

6. Selvi T, Sinha P, Subramaniam PM, Konapur PG, Prabha CV.A clinicopathological study of cholecystitis with special reference to analysis of cholelithiasis. International Journal of Basic Medical Science 2011; 2(2):68-72.

7. Awasthi N. A retrospective histopathological study of cholecystectomies. Int J Health Allied Sci. 2015;4:203-06.

8. Memon W, Khanzada TW, Samad A, Kumar B. Histopathological spectrum of gallbladder specimen after cholecystectomy. Pak J Med Sci 2011; 27:533-56.

9. Nigam M, Ranwaka R, Nigam B, Singh M, Devpura T P. Prevalance of carcinoma in symptomatic gallstone disease-A study following cholecystectomy. JEMDS 2013;25(2):4554-58.

10. Narendra GN, Gautam K. A spectrum of benign gallbladder diseases and their laparoscopic management: An experience of 100 patients. IJHRMLP 2015; 1(2): 25-31.

11. Arathi N, Awasthi S, Kumar A. Pathological profile of ${ }_{11}$ cholecystectomies at a teritiary centre. Natl J Med Dent Res. 2013;2(1):28-38.
12. Estrado RL, Brown NM, James CE: Chronic follicular cholecytitis: radiological, pathological and surgical aspects. Br J Surg 1960; 48:205.

13. Mohan H, Punia RP S, Dhawan SB, Ahal S, Sekhon MS. Morphological spectrum of gallstone disease in 1100 cholecystectomies in North India Indian Journal of Surgery, 2005; 67(3), 140-142

14. Sharma,Basu, Agarwal, Mishra. Gall Bladder disease in Gorakhpur region. Indian Journal Of Gastroenterology 1985:4(4):123-124

15. Guzman-Valdivia G. Xanthogranulomatous cholecystitis: 15 years Experience. World Journal of Surgery. 2004; 28(3):254-257.

16. Dixit VK, Parakash A, Gupta A et al. Xanthogranulatous Cholecystitis. Digestive Diseases and sciences 1998;43 (5) :122-125

17. Fox H, Mainwaring AR: Eosinophillic infiltration of gall bladder. Gastroenterology 1972; 63:1049- 1052.

18. Dabbs DJ. Eosinophilic and lymphoesinophilic cholecystitis. Am J SurgPathol 1993; 17: 497-501.

19. Durate I, Llanos O, Domke H, Harz C, Valdivieso V. Metaplasia and precursor lesions of gallbladder carcinoma. Frequency, distribution and probability of detection in routine histologic samples. Cancer 1993;72:1878-84.

20. Akritidis N, Mantzios G, Pappas G. Gallbladder adenomyomatosis presenting as fever of unknown origin: a case report. Hepatogastroenterology 2001; 48(37):112-

21. Indian Council for medical research (ICMR). Annual report of population based cancer registries of National Cancer Registry programme9 1996). New Delhi, ICMR publication, pg 18.

22. Pandey M, Pathak K, Gautam A et al. Carcinoma of the Gallbladder: A Retosepctive Review of 99 cases. Digestive Diseases and Sciences. 2001;46 (6): 1145-1151.

23. Esendagli G, Akarca FG, Balci S et al.A Retrospective Evaluation of the Epithelial Changes/Lesions and Neoplasms of the Gallbladder in Turkey and a Review of the Existing Sampling Methods:A Multicentre Study Turk Patoloji Derg 2018; 34:41-48.

24. Mondal B, Maulik D, Biswas BK, Sarkar GN, Ghosh D. Histopathological spectrum of gallstone disease from cholecystectomy specimen in rural areas of West Bengal, India- an approach of association between gallstone disease and gallbladder carcinoma. Int $\mathrm{J}$ Community Med Public Health 2016;3:3229-35.

25. Terada T. Histopathologic features and frequency of gall bladder lesions in consecutive 540 cholecystectomies. Int J Clin Exp Pathol 2013;6(1):91-96.

*Corresponding author:

Dr. Mangesh Londhe, Mailing address- plot no.3, Sainathnagar, Pune- 411044

Phone: +91 9850960153

Email: m.londhe03@gmail.com

Financial or other Competing Interests: None. 